\title{
INTEGRABILITY OF REVERSIBLE AND EQUIVARIANT QUADRATIC POLYNOMIAL DIFFERENTIAL SYSTEMS IN THE PLANE
}

\author{
JAUME LLIBRE AND CLAUDIA VALLS
}

\begin{abstract}
We study the existence of first integrals for the class of reversible and equivariant quadratic polynomial differential systems in the plane. We put special emphasis in the study of the analytic first integrals.
\end{abstract}

\section{INTRODUCTION}

For a planar differential system the existence of a first integral determines completely its phase portrait. However the explicit computation of first integrals is not an easy task. A first step is to compute those first integrals in different classes of functions such as the class of analytic functions.

These last years quadratic vector fields have been investigated intensively as one of the easiest families of nonlinear differential systems, and more than one thousand papers have been published about these vector fields (see for instance $[2,8,10,9]$ ), but the problem of classifying all the integrable quadratic vector fields remains open. For more information on the integrable differential vector fields in dimension 2 , see for instance [3].

The reversible and the equivariant differential equations have symmetries and this is important because a symmetry of a differential equation is a transformation that sends solutions to solutions simplifying the study of these differential systems. The equations describing a physical or a biological system often exhibit symmetries, therefore it is important to study such classes of systems. Reversible systems have an additional importance because reversibility has similar implications for the eigenvalues as in the Hamiltonian systems.

Let $\varphi: \mathbb{R}^{2} \rightarrow \mathbb{R}^{2}$ be an involution, that is, $\varphi \circ \varphi=\mathrm{Id}$. We say that a polynomial vector field $X$ is $\varphi$-reversible, if $X$ satisfies

$$
D \varphi(p) X(p)=-X \circ \varphi(p), \text { for all } p \in \mathbb{R}^{2},
$$

and we say that $X$ is $\varphi$-equivariant, if $X$ satisfies

$$
D \varphi(p) X(p)=X \circ \varphi(p) \text {, for all } p \in \mathbb{R}^{2} .
$$

In this paper we characterize the existence of analytic first integrals for all $\varphi$-reversible and $\varphi$-equivariant quadratic polynomial vector fields in the plane.

2010 Mathematics Subject Classification. Primary: 37J35, 34Cxx.

Key words and phrases. reversible, equivariant, quadratic planar polynomial equations, analytic first integral. 
It will be proved in section 2 that it is enough to consider the involution $\varphi(x, y)=(x,-y)$. This result is not new (see for instance [6]) but since it is easy to prove we show it here for completeness.

Since $\varphi(x, y)=(x,-y)$ it is easy to obtain that the $\varphi$-reversible quadratic polynomial vector fields in $\mathbb{R}^{2}$ have the form

$$
\begin{aligned}
& x^{\prime}=y\left(a_{0}+a_{1} x\right), \\
& y^{\prime}=b_{0}+b_{1} x+b_{2} x^{2}+b_{3} y^{2},
\end{aligned}
$$

where $a_{0}, a_{1}, b_{0}, b_{1}, b_{2}, b_{3} \in \mathbb{R}$, and that the $\varphi$-equivariant quadratic polynomial vector fields have the form

$$
\begin{aligned}
& x^{\prime}=a_{0}+a_{1} x+a_{2} x^{2}+a_{3} y^{2}, \\
& y^{\prime}=\left(b_{0}+b_{1} x\right) y,
\end{aligned}
$$

where $a_{0}, a_{1}, a_{2}, a_{3}, b_{0}, b_{1} \in \mathbb{R}$.

Let $U$ be an open subset of $\mathbb{R}^{2}$ such that $\mathbb{R}^{2} \backslash U$ has zero Lebesgue measure. A non-constant function $H: U \rightarrow \mathbb{R}$ is a first integral of the differential system (1) (respectively (2)) if it is constant on the solutions of system (1) (respectively (2)) contained in $U$. When a differential system has a first integral we say that it is integrable. We say that the first integral $H$ is analytic when the function $H$ is analytic in $U$ and in this case the system is called analytically integrable. When $U=\mathbb{R}^{2}$ we say that $H$ is a global analytic first integral.

The main objective of this paper is to study the existence of analytic first integrals for systems (1) and (2). We shall see that system (1) is always analytically integrable, see Theorem 1 . For system (2) we shall see that if $a_{3} b_{1}\left(a_{1} b_{1}-2 a_{2} b_{0}\right)=0$ then it is analytically integrable (see Theorems 2 , 3 and 4$)$. Finally in Theorem 5 we show that system (2) with $a_{3} b_{1}\left(a_{1} b_{1}-\right.$ $\left.2 a_{2} b_{0}\right) \neq 0$ is not globally analytically integrable.

Theorem 1. System (1) is analytically integrable. More precisely:

(a) If $a_{0}=a_{1}=0$, then a first integral is $x$.

(b) If $b_{0}=b_{1}=b_{2}=b_{3}=0$, then a first integral is $y$.

In the rest of the theorem we assume that $\left(a_{0}, a_{1}\right) \neq(0,0)$ and that $\left(b_{0}, b_{1}, b_{2}\right.$, $\left.b_{3}\right) \neq(0,0,0,0)$.

(c) If $b_{3}=0$ and $a_{1}=0$, then a first integral is

$$
y^{2}-\frac{x}{3 a_{0}}\left(6 b_{0}+3 b_{1} x+2 b_{2} x^{2}\right) .
$$

(d) If $b_{3}=0$ and $a_{1} \neq 0$, then a first integral is

$$
y^{2}-\frac{x\left(2 a_{1} b_{1}-2 a_{0} b_{2}+a_{1} b_{2} x\right)}{a_{1}^{2}}-\frac{G_{0}}{a_{1}^{3}} \log \left(a_{0}+a_{1} x\right),
$$

where $G_{0}=2\left(a_{1}^{2} b_{0}-a_{0} a_{1} b_{1}+a_{0}^{2} b_{2}\right)$.

(e) If $b_{3} \neq 0$ and $a_{1}=0$, then a first integral is

$$
\exp \left(-\frac{2 b_{3}}{a_{0}} x\right)\left(2 b_{3}^{3} y^{2}+a_{0}^{2} b_{2}+a_{0} b_{3}\left(b_{1}+2 b_{2} x\right)+2 b_{3}^{2}\left(b_{0}+b_{1} x+b_{2} x^{2}\right)\right. \text {. }
$$


(f) If $b_{3} a_{1} \neq 0$ and $\left(b_{3}-a_{1}\right)\left(2 b_{3}-a_{1}\right) \neq 0$, then a first integral is

$$
\left(a_{0}+a_{1} x\right)^{-\frac{2 b_{3}}{a_{1}}}\left[y^{2}-\frac{G_{1}}{b_{3}\left(b_{3}-a_{1}\right)\left(2 b_{3}-a_{1}\right)}\right],
$$

where $G_{1}=-a_{1}^{2} b_{0}-a_{0}^{2} b_{2}-a_{0} b_{3}\left(b_{1}+2 b_{2} x\right)-2 b_{3}^{2}\left(b_{0}+b_{1} x+b_{2} x^{2}\right)+$ $a_{1}\left(a_{0} b_{1}+3 b_{0} b_{3}+2 b_{1} b_{3} x+b_{2} b_{3} x^{2}\right)$.

(g) If $b_{3} a_{1} \neq 0, b_{3}=a_{1}$ and $b_{2}=0$, then a first integral is

$$
\frac{1}{\left(a_{0}+a_{1} x\right)^{2}}\left[y^{2}+\frac{a_{1} b_{0}+a_{0} b_{1}+2 a_{1} b_{1} x}{a_{1}^{2}}\right] \text {. }
$$

(h) If $b_{3} a_{1} \neq 0, b_{3}=a_{1}$ and $b_{2} \neq 0$, then a first integral is

$$
\frac{1}{\left(a_{0}+a_{1} x\right)^{2}}\left[a_{1}^{3} y^{2}-G_{2}-2 b_{2}\left(a_{0}+a_{1} x\right)^{2} \log \left(a_{0}+a_{1} x\right)\right],
$$

where $G_{2}=3 a_{0}^{2} b_{2}-a_{1}^{2}\left(b_{0}+2 b_{1} x\right)-a_{0} a_{1}\left(b_{1}-4 b_{2} x\right)$.

(i) If $b_{3} a_{1} \neq 0, b_{3}=a_{1} / 2$ and $a_{1} b_{1}=2 a_{0} b_{2}$, then a first integral is

$$
\frac{1}{a_{0}+a_{1} x}\left[a_{1}^{3} y^{2}-2\left(a_{0}^{2} b_{2}+a_{0} a_{1} b_{2} x+a_{1}^{2}\left(-b_{0}+b_{2} x^{2}\right)\right)\right] .
$$

(j) If $b_{3} a_{1} \neq 0, b_{3}=a_{1} / 2$ and $a_{1} b_{1} \neq 2 a_{0} b_{2}$, then a first integral is

$$
\begin{gathered}
\frac{1}{a_{0}+a_{1} x}\left[a_{1}^{3} y^{2}-G_{3}-2\left(a_{1} b_{1}-2 a_{0} b_{2}\right)\left(a_{0}+a_{1} x\right) \log \left(a_{0}+a_{1} x\right)\right], \\
\text { where } G_{3}=-2\left(a_{1}^{2} b_{0}-a_{0} a_{1} b_{1}+a_{0}^{2} b_{2}-a_{0} a_{1} b_{2} x-a_{1}^{2} b_{2} x^{2}\right) .
\end{gathered}
$$

Theorem 2. System (2) with condition $a_{3}=0$ is analytically integrable. More precisely:

(a) If $a_{0}=a_{1}=a_{2}=0$, then a first integral is $x$.

(b) If $b_{0}=b_{1}=0$, then a first integral is $y$.

In the rest of the theorem we assume that $\left(a_{0}, a_{1}, a_{2}\right) \neq(0,0,0)$ and that $\left(b_{0}, b_{1}\right) \neq(0,0)$.

(c) If $a_{1}=a_{2}=0$, then a first integral is

$$
\log y-\frac{x}{2 a_{0}}\left(2 b_{0}+b_{1} x\right) .
$$

(d) If $a_{2}=0$ and $a_{1} \neq 0$, then a first integral is

$$
\log y-\frac{a_{1} b_{0}-a_{0} b_{1}}{a_{1}^{2}} \log \left(a_{0}+a_{1} x\right)-\frac{b_{1}}{a_{1}} x .
$$

(e) If $a_{2} \neq 0$ and $a_{0}=a_{1}^{2} /\left(4 a_{2}\right)$, then a first integral is

$$
\log y-\frac{b_{1}}{a_{2}} \log \left(a_{1}+2 a_{2} x\right)+\frac{2 a_{2} b_{0}-a_{1} b_{1}}{a_{2}\left(a_{1}+2 a_{2} x\right)} .
$$

(f) If $a_{2} \neq 0$ and $a_{0} \neq a_{1}^{2} /\left(4 a_{2}\right)$, then a first integral is

$$
\log y-\frac{b_{1}}{2 a_{2}} \log \left(a_{0}+x\left(a_{1}+a_{2} x\right)\right)+\frac{a_{1} b_{1}-2 a_{2} b_{0}}{a_{2} \sqrt{4 a_{0} a_{2}-a_{1}^{2}}} \arctan \left(\frac{a_{1}+2 a_{2} x}{\sqrt{4 a_{0} a_{2}-a_{1}^{2}}}\right) .
$$


Theorem 3. System (2) with conditions $a_{3} \neq 0$ and $b_{1}=0$ is integrable with first integral $y$ if $b_{0}=0$, and

$$
\frac{\left(-a_{1}-2 a_{2} x\right) Y_{S_{1}}\left(S_{2} y\right)+\sqrt{a_{2}} \sqrt{a_{3}} y\left(Y_{S_{1}+1}\left(S_{2} y\right)-Y_{S_{1}-1}\left(S_{2} y\right)\right)}{\left(a_{1}+2 a_{2} x\right) J_{S_{1}}\left(S_{2} y\right)+\sqrt{a_{2}} \sqrt{a_{3}} y\left(J_{S_{1}-1}\left(S_{2} y\right)-J_{S_{1}+1}\left(S_{2} y\right)\right)},
$$

if $b_{0} \neq 0$, where $S_{1}=\sqrt{a_{1}^{2}-4 a_{0} a_{2}} /\left(2 b_{0}\right), S_{2}=\sqrt{a_{2} a_{3}} / b_{0}, Y_{a}(z)$ is the Bessel function of the second kind, and $J_{a}(z)$ is the Bessel function of the first kind.

For more details on the Bessel functions see [1].

Theorem 4. System (2) with conditions $a_{3} b_{1} \neq 0$, and $a_{1} b_{1}=2 a_{2} b_{0}$ is analytically integrable. More precisely:

(a) If $a_{2}=0$, then a first integral is

$$
\left(b_{0}+b_{1} x\right)^{2}-a_{3} b_{1} y^{2}-\frac{2}{b_{1}}\left(a_{0} b_{1}^{2}-a_{2} b_{0}^{2}\right) \log y .
$$

(b) If $a_{2}=b_{1}$, then a first integral is

$$
\frac{1}{y^{2}}\left[a_{0} b_{1}^{2}-a_{2} b_{0}^{2}+b_{1}\left(b_{0}+b_{1} x\right)^{2}-2 a_{3} b_{1}^{2} y^{2} \log y\right] .
$$

(c) If $a_{2}\left(a_{2}-b_{1}\right) \neq 0$, then a first integral is

$$
y^{-\frac{2 a_{2}}{b_{1}}}\left[a_{0} b_{1}\left(a_{2}-b_{1}\right)+2 a_{2} b_{0}\left(a_{2}-b_{1}\right) x+a_{2} b_{1}\left(a_{2}-b_{1}\right) x^{2}+a_{2} a_{3} b_{1} y^{2}\right] \text {. }
$$

Theorem 5. System (2) with $a_{3} b_{1}\left(a_{1} b_{1}-2 a_{2} b_{0}\right) \neq 0$ has no global analytic first integrals.

Theorems 1, 2, 3, 4 and 5 are proved in sections $4,5,6,7$ and 8 , respectively.

\section{REVERSIBLE AND EQUIVARIANT QUADRATIC POLYNOMIAL VECTOR FIELDS}

We show in this section that in the definition of reversible and equivariant quadratic polynomial vector fields we can always consider that the involution $\varphi$ is given by $\varphi(x, y)=(x,-y)$. More precisely, we will prove the following result.

Proposition 1. Let $\varphi: \mathbb{R}^{2} \rightarrow \mathbb{R}^{2}$ be a polynomial involution, and let $X$ be a $\varphi$-reversible (respectively $\varphi$-equivariant) polynomial vector field of degree $m>1$ in $\mathbb{R}^{2}$. Then $\varphi$ is conjugated to $\psi$ given by $\psi=\operatorname{diag}(1,-1)$.

Proof. We will prove the proposition only for the case in which $X$ is a $\varphi$ reversible polynomial vector field, since the case in which $X$ is $\varphi$-equivariant is completely analogous. We first show that under the assumptions, $\varphi$ is linear. Let $q$ be the degree of the polynomial involution $\varphi: \mathbb{R}^{2} \rightarrow \mathbb{R}^{2}$. We will show that $q=1$. Indeed since $X$ is a $\varphi$-reversible vector field, we have that $D \varphi(p) X(p)=-X(\varphi(p))$. This equation implies that if $m q=q-1+m$, or equivalently $q(m-1)=m-1$. Taking into account that $m \neq 1$ we conclude that $q=1$. Therefore, since $\varphi$ is linear and it is an involution we get that det $\varphi= \pm 1$. By the Jordan's normal form theorem, there is a linear change of variables $h: \mathbb{R}^{2} \rightarrow \mathbb{R}^{2}$ such that $\psi=h^{-1} \varphi h$ where $\psi$ is formed by 
not zero eigenvalues $\lambda_{1}$ and $\lambda_{2}$ satisfying $\lambda_{1} \lambda_{2}= \pm 1$. Furthermore, using that $\psi^{2}=$ Id we conclude that $\lambda_{1}= \pm 1$ and $\lambda_{2}= \pm 1$. Hence we can always assume that $\lambda_{1}=1$ and $\lambda_{2}=-1$. This concludes the proof.

\section{Preliminary Results}

The vector field $X$ associated to the system

$$
x^{\prime}=P(x, y), \quad y^{\prime}=Q(x, y),
$$

where $P$ and $Q$ are real polynomials in the variables $x, y$ is defined by

$$
X=P(x, y) \frac{\partial}{\partial x}+Q(x, y) \frac{\partial}{\partial y} .
$$

We introduce the following auxiliary result due to Poincaré in [7], see also [4] for a direct proof. Through the paper $\mathbb{Z}^{+}$will denote the set of non-negative integers.

Theorem 6. Assume that the eigenvalues $\lambda_{1} \neq 0$ and $\lambda_{2} \neq 0$ at some singular point $p$ of $X$ do not satisfy any resonance condition of the form

$$
\lambda_{1} k_{1}+\lambda_{2} k_{2}=0 \quad \text { for } k_{1}, k_{2} \in \mathbb{Z}^{+} \text {with } k_{1}+k_{2}>0 .
$$

Then system (19) has no analytic first integrals defined in a neighborhood of $p$.

The following result is due to Li, Llibre and Zhang, see [5].

Theorem 7. Assume that the eigenvalues $\lambda_{1}$ and $\lambda_{2}$ at some singular point $p$ of $X$ satisfy that $\lambda_{1}=0$ and $\lambda_{2} \neq 0$. Then system (19) has no analytic first integrals in a neighborhood of $p$ if $p$ is isolated in the set of all singular points of $X$.

\section{Proof of Theorem 1}

It is clear that if $a_{0}=a_{1}=0$ then $x$ is a polynomial first integral, and if $b_{0}=b_{1}=b_{2}=b_{3}=0$, then $y$ is a polynomial first integral. So statements (a) and (b) are proved. $0)$.

From now on we assume that $\left(a_{0}, a_{1}\right) \neq(0,0)$ and $\left(b_{0}, b_{1}, b_{2}, b_{3}\right) \neq(0,0,0$,

We rewrite systems (1) into the form

$$
\frac{d y}{d x}=\frac{b_{0}+b_{1} x+b_{2} x^{2}}{y\left(a_{0}+a_{1} x\right)}+\frac{b_{3} y}{a_{0}+a_{1} x} .
$$

Now taking the variable $v=y^{2}$ we get that the above system becomes the following linear differential system in the variable $v$,

$$
v^{\prime}=\frac{d v}{d x}=\frac{2 b_{3}}{a_{0}+a_{1} x} v+\frac{2\left(b_{0}+b_{1} x+b_{2} x^{2}\right)}{a_{0}+a_{1} x} .
$$

We consider eight cases which correspond with the eight statements $(c)-(j)$ of the theorem.

(c) $b_{3}=0$ and $a_{1}=0$. In this case the general solution of system (20) is

$$
v(x)=\frac{x\left(6 b_{0}+3 b_{1} x+2 b_{2} x^{2}\right)}{3 a_{0}}+H,
$$


where $H$ is an integration constant. Hence system (1) under these assumptions is integrable with the first integral (3).

(d) $b_{3}=0$ and $a_{1} \neq 0$. In this case this case the general solution of system (20) is

$$
v(x)=\frac{x\left(2 a_{1} b_{1}-2 a_{0} b_{2}+a_{1} b_{2} x\right)}{a_{1}^{2}}+H+\frac{G_{0}}{a_{1}^{3}} \log \left(a_{0}+a_{1} x\right),
$$

where $H$ is an integration constant. Hence system (1) under these assumptions is integrable with the first integral (4).

(e) $b_{3} \neq 0$ and $a_{1}=0$. In this case the general solution of system (20) is

$2 b_{3}^{3} v(x)=-a_{0}^{2} b_{2}-a_{0} b_{3}\left(b_{1}+2 b_{2} x\right)-2 b_{3}^{2}\left(b_{0}+x\left(b_{1}+b_{2} x\right)-b_{3} \exp \left(\frac{2 b_{3} x}{a_{0}}\right) H\right)$,

where $H$ is a first integral. Hence system (1) is integrable with the first integral (5).

(f) $b_{3} a_{1} \neq 0$ and $\left(b_{3}-a_{1}\right)\left(2 b_{3}-a_{1}\right) \neq 0$. The general solution of system (20) is

$$
v(x)=\frac{G_{1}}{b_{3}\left(b_{3}-a_{1}\right)\left(2 b_{3}-a_{1}\right)}+\left(a_{0}+a_{1} x\right)^{2 b_{3} / a_{1}} H,
$$

where $H$ is a first integral. Hence system (1) has the first integral (6).

(g) $b_{3} a_{1} \neq 0, b_{3}=a_{1}$ and $b_{2}=0$. The general solution of system (20) is

$$
v(x)=-\frac{a_{1} b_{0}+a_{0} b_{1}+2 a_{1} b_{1} x}{a_{1}^{2}}+\left(a_{0}+a_{1} x\right)^{2} H .
$$

Hence system (1) has the first integral (7).

(h) $b_{3} a_{1} \neq 0, b_{3}=a_{1}$ and $b_{2} \neq 0$. The general solution of system (20) is

$$
a_{1}^{3} v(x)=G_{2}+a_{1}^{3}\left(a_{0}+a_{1} x\right)^{2} H+2 b_{2}\left(a_{0}+a_{1} x\right)^{2} \log \left(a_{0}+a_{1} x\right) .
$$

Hence system (1) has the first integral (8).

(i) $b_{3} a_{1} \neq 0, b_{3}=a_{1} / 2$ and $a_{1} b_{1}=2 a_{0} b_{2}$. In this case the general solution of system (2) is

$$
v(x)=2\left(\frac{a_{0}^{2} b_{2}+a_{0} a_{1} b_{2} x+a_{1}^{2}\left(-b_{0}+b_{2} x^{2}\right)}{a_{1}^{3}}\right)+\left(a_{0}+a_{1} x\right) H .
$$

Hence system (1) has the first integral (9).

(j) $b_{3} a_{1} \neq 0, b_{3}=a_{1} / 2$ and $a_{1} b_{1} \neq 2 a_{0} b_{2}$. In this case the general solution of system (20) is

$$
a_{1}^{3} v(x)=G_{3}+a_{1}^{3}\left(a_{0}+a_{1} x\right) H+2\left(a_{1} b_{1}-2 a_{0} b_{2}\right)\left(a_{0}+a_{1} x\right) \log \left(a_{0}+a_{1} x\right) .
$$

Hence system (1) has the first integral (10).

\section{Proof of Theorem 2}

We consider system (2) with $a_{3}=0$. If $a_{0}=a_{1}=a_{2}=0$ then $x$ is a polynomial first integral, and if $b_{0}=b_{1}=0$ then $y$ is a polynomial first integral. Therefore statements (a) and (b) hold. 
From now on we assume that $\left(a_{0}, a_{1}, a_{2}\right) \neq(0,0,0)$ and that $\left(b_{0}, b_{1}\right) \neq$ $(0,0)$. In this case we write system $(2)$ in the form

$$
\frac{d x}{d y}=\frac{a_{0}+a_{1} x+a_{2} x^{2}}{y\left(b_{0}+b_{1} x\right)} .
$$

It is clear that a first integral of system (21) is

$$
H=\log y-\int \frac{b_{0}+b_{1} x}{a_{0}+a_{1} x+a_{2} x^{2}} d x .
$$

Now we consider four cases which correspond with the four statements (c)-(f) of the theorem.

(c) $a_{1}=a_{2}=0$. In this case, from system (22) we get that $H$ given in (11) is a first integral.

(d) $a_{2}=0$ and $a_{1} \neq 0$. Again from (22) we get that $H$ given in (12) is a first integral.

(e) $a_{2} \neq 0$ and $a_{0}=a_{1}^{2} /\left(4 a_{2}\right)$. From system (22) we obtain that $H$ given in (13) is a first integral.

(f) $a_{2} \neq 0$ and $a_{0} \neq a_{1}^{2} /\left(4 a_{2}\right)$. In this case $H$ given in (14) is a first integral.

\section{Proof of Theorem 3}

Here $b_{1}=0$. When $b_{0}=0$ it is clear that $y$ is a polynomial first integral. Now we assume that $b_{0} \neq 0$. In this case we write system (2) in the form

$$
x^{\prime}(y)=\frac{d x}{d y}=\frac{a_{0}+a_{1} x+a_{2} x^{2}+a_{3} y^{2}}{b_{0} y} .
$$

Solving it we get $x(y)=N / D$ where

$$
\begin{aligned}
N= & -\sqrt{a_{2} a_{3}} H y J_{S_{1}-1}\left(S_{2} y\right)-a_{1} H J_{S_{1}}\left(S_{2} y\right)-a_{1} Y_{S_{1}}\left(S_{2} y\right) \\
& +\sqrt{a_{2} a_{3}} y\left(H J_{S_{1}+1}\left(S_{2} y\right)-Y_{S_{1}-1}\left(S_{2} y\right)+Y_{S_{1}+1}\left(S_{2} y\right)\right), \\
D= & 2 a_{2}\left(H J_{S_{1}}\left(S_{2} y\right)+Y_{S_{1}}\left(S_{2} y\right)\right),
\end{aligned}
$$

and where $H$ is a first integral. Hence system (2) is integrable with the first integral (15).

\section{Proof of Theorem 4}

We introduce the change of variables (recall that $a_{3} b_{1} \neq 0$ )

$$
X=b_{0}+b_{1} x, \quad Y=y \quad \text { with inverse change } \quad x=\frac{X-b_{0}}{b_{1}}, \quad y=Y .
$$

With this change of variables, system (2) becomes

$$
\begin{aligned}
& X^{\prime}=A_{0}+A_{1} X+A_{2} X^{2}+A_{3} Y^{2}, \\
& Y^{\prime}=X Y
\end{aligned}
$$

where

$$
A_{0}=a_{0} b_{1}-a_{1} b_{0}+\frac{a_{2} b_{0}^{2}}{b_{1}}, \quad A_{1}=a_{1}-\frac{2 a_{2} b_{0}}{b_{1}}, \quad A_{2}=\frac{a_{2}}{b_{1}}, \quad A_{3}=a_{3} b_{1} .
$$


Under the assumptions of the theorem, we can rewrite system (23) of the form

where

$$
\frac{d X}{d Y}=\frac{A_{0}+A_{3} Y^{2}}{X Y}+\frac{A_{2} X}{Y},
$$

$$
A_{0}=\frac{a_{0} b_{1}^{2}-a_{2} b_{0}^{2}}{b_{1}}, \quad A_{2}=\frac{a_{2}}{b_{1}}, \quad A_{3}=a_{3} b_{1} .
$$

We consider three cases which correspond with the three statements (a)-(c) of the theorem.

(a) $a_{2}=0$. In this case $A_{2}=0$. Computing the general solution of system (24) and isolating the integration constant $H$ we obtain

$$
H=X^{2}-A_{3} Y^{2}-2 A_{0} \log Y,
$$

which in the original variables is the integral in (16).

(b) $a_{2}=b_{1}$. In this case $A_{2}=1$. Again computing the general solution of system (24) and isolating the integration constant $H$ we obtain

$$
H=\frac{1}{Y^{2}}\left[A_{0}+X^{2}-2 A_{3} Y^{2} \log Y\right]
$$

which in the original variables is the integral in (17).

(c) $a_{2}\left(a_{2}-b_{1}\right) \neq 0$. In this case we have $A_{2}\left(A_{2}-1\right) \neq 0$. The integration constant $H$ of the general solution of system (24) is

$$
H=Y^{-2 A_{2}}\left[-A_{0}+A_{0} A_{2}-A_{2} X^{2}+A_{2}^{2} X^{2}+A_{2} A_{3} Y^{2}\right],
$$

which in the original variables is the integral in (18).

\section{Proof of Theorem 5}

We work with system (23). We consider four different cases.

Case 1: $A_{0}=0$. In this case the origin is a singular point. Its eigenvalues are 0 and $A_{1} \neq 0$. Since this singular point is isolated (because $A_{3} \neq 0$ ), Theorem 7 implies that system (23) has no analytic first integrals.

Case 2: $A_{0} \neq 0$ and $A_{2}=0$. System (23) has the singular points

$\left(\bar{X}_{1}, \bar{Y}_{1}\right)=\left(0, \sqrt{-\frac{A_{0}}{A_{3}}}\right),\left(\bar{X}_{2}, \bar{Y}_{2}\right)=\left(0,-\sqrt{-\frac{A_{0}}{A_{3}}}\right),\left(\bar{X}_{3}, \bar{Y}_{3}\right)=\left(-\frac{A_{0}}{A_{1}}, 0\right)$.

The eigenvalues of $\left(\bar{X}_{3}, \bar{Y}_{3}\right)$ are $A_{1}$ and $-A_{0} / A_{1}$. Suppose that there exists $k_{1}, k_{2} \in \mathbb{Z}^{+}$such that $k_{1} \lambda_{1}+k_{2} \lambda_{2}=0$. Note that by Theorem 6 if such integers do not exist the theorem is proved. Then $\lambda_{1}=-\alpha \lambda_{2}$ with $\alpha$ a positive rational, and hence in particular $\lambda_{1} \lambda_{2}=-\alpha \lambda_{2}^{2}<0$. Since $\lambda_{1} \lambda_{2}=$ $-A_{0}$ we must have that $A_{0}$ is positive.

Moreover, the eigenvalues $\lambda_{1}$ and $\lambda_{2}$ of $\left(\bar{X}_{1}, \bar{Y}_{1}\right)$ are $\left(A_{1} \pm \sqrt{A_{1}^{2}-8 A_{0}}\right) / 2$. Again we must have that $\lambda_{1} \lambda_{2}<0$, otherwise the theorem holds by Theorem 6. Note that $\lambda_{1} \lambda_{2}=2 A_{0}>0$, and then we cannot have $k_{1} \lambda_{1}+k_{2} \lambda_{2}=0$. The theorem is proved in this case.

Case 3: $A_{0} A_{2} \neq 0$ and $A_{2}=A_{1}^{2} /\left(4 A_{0}\right)$. In this case $(\bar{X}, \bar{Y})=\left(-2 A_{0} / A_{1}, 0\right)$ is a singular point. Its eigenvalues are 0 and $-2 A_{0} / A_{1} \neq 0$. Since this singular point is isolated (because $A_{3} \neq 0$ ), Theorem 7 implies that system (23) has no analytic first integrals. 
Case 4: $A_{0} A_{2}\left(A_{1}^{2}-4 A_{0} A_{2}\right) \neq 0$. We assume that $H=H(X, Y)$ is a global analytic first integral in a neighborhood of $Y=0$. We write it as

$$
H=\sum_{k \geq 0} H_{k}(X) Y^{k}
$$

where each $H_{k}$ is an analytic function in the variable $X$. Without loss of generality we can assume that $H$ has no constant term, i.e. $H_{0}(0)=0$. We will show by induction that

$$
H_{k}=0 \quad \text { for } k \geq 0 \text {. }
$$

Then clearly from (25) we will obtain that system (23) has no global analytic first integrals, and the proof of the theorem is done.

Since $H$ is a first integral of system (23) it must satisfy

$$
\left(A_{0}+A_{1} X+A_{2} X^{2}+A_{5} Y^{2}\right) \sum_{k \geq 0} H_{k}^{\prime}(X) Y^{k}+\sum_{k \geq 0} k X H_{k}(X) Y^{k}=0 .
$$

Computing the terms of degree 0 in $Y$ we get that $H_{0}^{\prime}(X)=0$, that is, $H_{0}$ is a constant. Since $H$ has no constant term we obtain that $H_{0}=0$ which proves (26) for $k=0$.

Now assume that (26) is true for $k=0, \ldots, \ell-1$ and we will show it for $k=\ell$. We have that $H=Y^{\ell} \sum_{k \geq 0} H_{k+\ell} Y^{k}$. Computing the terms of degree $\ell$ in the variable $Y$ in (27) we get that

$$
\left(A_{0}+A_{1} X+A_{2} X^{2}\right) H_{\ell}^{\prime}(X)+\ell X H_{\ell}(X)=0 .
$$

Solving this linear equation we get

$$
H_{\ell}(X)=K\left(A_{0}+A_{1} X+A_{2} X^{2}\right)^{-\frac{\ell}{2 A_{2}}} \exp \left(\frac{A_{1} \ell}{A_{2} \tilde{A}} \arctan \left(\frac{A_{1}+2 A_{2} X}{\tilde{A}}\right)\right),
$$

where $\tilde{A}=\sqrt{4 A_{0} A_{2}-A_{1}^{2}}$ and $K \in \mathbb{R}$. Since $H_{\ell}$ must be a global analytic function in $X$, we get that $K=0$ and thus $H_{\ell}(X)=0$. This ends the induction process and the proof of the theorem is completed.

\section{ACKNOWLEDGMENTS}

The first author has been supported by the grants MICIIN/FEDER MTM 2009-03437, AGAUR 2009SGR 410 and ICREA Academia. The second author has been partially supported by FCT through CAMGSD.

\section{REFERENCES}

[1] M. Abramowitz And I.A. Stegun, Handbook of mathematical functions with formulas, graphs, and mathematical tables, National Bureau of Standards Applied Mathematics Series, 55, Washington, D.C., 1964.

[2] L. CAIRó AND J. LliBRe, Phase portraits of planar semi-homogeneous systems I, Nonlinear Anal. 29 (1997), 783-811.

[3] J. Chavarriga, H. Giacomini, J. Giné and J. Llibre, On the integrability of two-dimensional flows, J. Differential Equations 157 (1999), 163-182.

[4] S. D. FurTa, On non-integrability of general systems of differential equations, Z. Angew. Math. Phys. 47 (1996), 112-131.

[5] W. Li, J. Llibre And X. ZhAng, Local first integrals of differential systems and diffeomorphisms, Z. Angew. Math. Phys. 54 (2003), 1-21.

[6] J. Llibre AND J.C. MedRAdo, Darboux integrability and reversible quadratic vector fields, Rocky Mountain J. Math. 35 (2005), 1999-2057. 
[7] H. Poincaré, Mémoire sur les courbes définies par les équations différentielles (Oeuvreus de Henri Poincaré, I), Gauthiers-Villars, Paris, 1951, pp. 95-114.

[8] J. W. REYN, A bibliography of the qualitative theory of quadratic systems of differential equations in the plane, Delf University of Technology, http://ta.twi.tudelft.nl/DV/Staff/J.W.Reyn.html, 1997.

[9] Y. YAnqIAn And Others, Theory of Limit Cycles, Transl. Math. Monographs 66, Amer. Math. Soc., Providence, 1984.

[10] Y. YAnqIan, Qualitative Theory of Polynomial Differential Systems, Shanghai Scientific \& Technical Publishers, Shanghai, 1995 (in Chinese).

Departament de Matemàtiques, Universitat Autònoma de Barcelona, 08193 Bellaterra, Barcelona, Catalonia, Spain

E-mail address: jllibre@mat.uab.cat

Departamento de Matemática, Instituto Superior Técnico, Universidade TÉCNica DE LisboA, 1049-001 Lisboa, Portugal

E-mail address: cvalls@math.ist.utl.pt 